Arch. hist. jap. Vol. 17, n. 3 (July 1959).

P. $459-473$.

2nd Dept. of Anat., Wakayama Med. College (Director: Prof. K. FUJIE).

\title{
Studies on the Effects of Amino-Acid on the Secretory Activity of the Gastric Peptic Cells and the Pancreatic Cells.
}

II. On the Effects of Amino-Acid in Diet.

アミノ酸が胃腺主細胞及び膵細胞の分泌機能に及ぼす影響について.

II. 食餌中のアミ, 酸の效果.

Michihiro NISHIOKA 西岡道弘.

(Received April 20,1959.)

The author, in a former report, studied the effects of amino-acid solution or emulsion injected into the stomach on the secretory activity of the gastric peptic and the pancreatic cells, and found that the most effective amino-acid was Histidine, and the ones less effective than Histidine to be Methionine, Tyrosine and Tryptophane. The effects of the others almost negligible. These four amino-acids had the potency to get the gastric surface cells to discharge productin into the blood.

The reason why the author tested aminoacids was due to the findings of TOJYO viz. that the inhibited secretion of productin and the low secretory activity of the gastric peptic cells, resulting from a lack of protein in diet, were prevented by a micro-dose of Histidine in a non-protein diet. Therefore the author attempted to prove here, whether the above effective amino-acids were also effective when their micro-dose was given with the non-protein diet.

\section{Materials and Methods.}

Adult and male rats, weighting $100-120 \mathrm{~g}$, accustomed to a regular feeding of Mc COLLUM's artificial diet were used. They were fed for 10 days with the artificial diet from which protein was removed and, instead of it, $5 \mathrm{mg}$ (daily per rat) each of Peptone, Methionine, Histidine, Tyrosine, Tryptophane or Histamine was given (Table 1) - described below as the Peptone-diet, the Methionine-diet, the Histidine-diet, etc. On the 11 th day, samples were taken at periods 1 . when rats were still unfed, 2. $\frac{1}{2}, 1,1 \frac{1}{2}, 2$, and $3 \mathrm{hrs}$. after administration of diet.

Table 1. Description of the diet (per rat, daily) (g).

\begin{tabular}{|c|c|c|c|c|c|c|c|c|c|}
\hline & 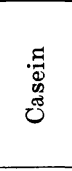 & 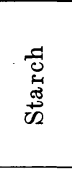 & 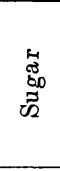 & 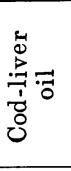 & 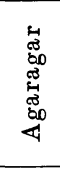 & 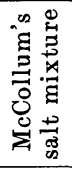 & 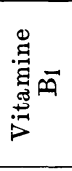 & 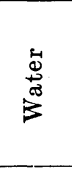 & 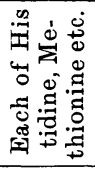 \\
\hline $\begin{array}{c}\text { Mc COLLUM's artificial } \\
\text { diet }\end{array}$ & 1.47 & 6.08 & 1.0 & 1.0 & 0.2 & 0.2 & 0.05 & 10.0 & \\
\hline $\begin{array}{l}\text { The experimental } \\
\text { diet }\end{array}$ & 0.0 & 7.55 & 1.0 & 1.0 & 0.2 & 0.2 & 0.05 & 10.0 & 0.005 \\
\hline
\end{tabular}


To take samples, LUNA's liquid was injected into all arteries through the left heart ventricle for the vital-fixation under deep ether narcosis. Small pieces were taken from the glandular portion of the stomach and from the pancreas, and they were placed in KOLSTER's liquid for the post-fixation. Paraffiae sections of $4 \mu$ were made and they were stained by HEIDENHAIN's iron haematoxylin. The gastric peptic cells, the surface cells of the gastric mucous membrane and the pancreatic cells were observed.

\section{Observations.}

In the gastric peptic cells, the quantity of secretion granules and of secretion vacuoles is described in the table with the form of plastosomes (pls.) and the aspect of the gland lumen. In the gastric surface cells, the quantity of productin vacuoles (FUJIE) in the cell, in the pancreatic cells, the quantity, the distribution and the size-irregularity of zymogen granules in the cell, the form of plastosomes and the aspect of the gland lumen are tabulated. These observations are described in the same manner as in former reports (Arch. hist. jap. 17, 1959).

A. The gastric peptic cells and the gastric surface cells. (Table 2-13)

Table 2. The Peptone-diet for 10 days. The peptic cells.

\begin{tabular}{|c|c|c|c|c|c|c|}
\hline \multirow{3}{*}{ Hrs. } & \multicolumn{3}{|c|}{ Total result } & \multirow{2}{*}{\multicolumn{2}{|c|}{$\begin{array}{l}\text { Representative } \\
\text { result }\end{array}$}} & \multirow{3}{*}{$\begin{array}{l}\text { Gland } \\
\text { lumen }\end{array}$} \\
\hline & \multicolumn{2}{|c|}{$\begin{array}{l}\text { Quantity of the intracel- } \\
\text { lular secretory substance }\end{array}$} & \multirow{2}{*}{$\begin{array}{l}\text { Form of } \\
\text { pls. }\end{array}$} & & & \\
\hline & Granules & Vacuoles & & Granules & Vacuoles & \\
\hline Before & $-\sim+$ & $-\sim+$ & $\begin{array}{l}\text { long rod, } \\
\text { short rod }\end{array}$ & $-\sim+$ & $-\sim+$ & $\begin{array}{c}\text { a little wide, } \\
\text { narrow }\end{array}$ \\
\hline$\frac{1}{2}$ & $\pm \sim++$ & $\pm \sim++$ & $\begin{array}{l}\text { short rod, } \\
\text { guitar form, } \\
\text { long rod, } \\
\text { supra-short } \\
\text { rod }\end{array}$ & $\begin{array}{l}+\sim++ \\
+\sim++ \\
\pm \sim+\end{array}$ & $\begin{array}{c} \pm \sim+ \\
+\sim++ \\
\pm\end{array}$ & $\begin{array}{l}\text { a little wide, } \\
\text { narrow }\end{array}$ \\
\hline 1 & $\pm \sim+++$ & $\pm \sim++$ & $\begin{array}{l}\text { short rod, } \\
\text { supra-short } \\
\text { rod, rosary } \\
\text { form, guitar } \\
\text { form }\end{array}$ & $+\sim++$ & $\pm \sim+(+)$ & $\begin{array}{l}\text { a little wide, } \\
\text { (wide) }\end{array}$ \\
\hline $1 \frac{1}{2}$ & $-\sim+++$ & $-\sim++$ & $\begin{array}{l}\text { short rod, } \\
\text { rosary form, } \\
\text { guitar form, } \\
\text { long rod }\end{array}$ & $+\sim++$ & $\pm \sim++$ & $\begin{array}{l}\text { a little wide, } \\
\text { wide }\end{array}$ \\
\hline 2 & $-\sim++$ & $\pm \sim++$ & $\begin{array}{l}\text { short or long } \\
\text { rod, guitar } \\
\text { form }\end{array}$ & $+\sim+(+)$ & $\pm=-+$ & $\begin{array}{l}\text { a little wide, } \\
\text { narrow }\end{array}$ \\
\hline 3 & $\cdots \sim++$ & $\pm \sim+++$ & $\begin{array}{c}\text { long rod, } \\
\text { short rod, } \\
\text { supra-short } \\
\text { rod }\end{array}$ & $\begin{array}{l}+\sim++ \\
-\sim+\end{array}$ & $\begin{array}{c} \pm \sim+ \\
+t \sim+t+\end{array}$ & $\begin{array}{l}\text { a little wide; } \\
\text { narrow }\end{array}$ \\
\hline
\end{tabular}


Table 3. The Peptone-diet for 10 days. The productin vacuoles in the gastric surface cells.

\begin{tabular}{|c|c|c|c|c|c|c|}
\hline & \multicolumn{6}{|c|}{ Hrs. } \\
\hline & Before & $\frac{1}{2}$ & 1 & $1 \frac{1}{2}$ & 2 & 3 \\
\hline Total result & $+\sim+++$ & $-\sim++$ & $-\sim+t$ & $-\sim+t$ & $\pm \sim+t+$ & $\pm \sim+1+$ \\
\hline $\begin{array}{c}\text { Representative } \\
\text { result }\end{array}$ & $t \sim+t-t$ & $-\underset{(+)}{\sim+}$ & $\pm \sim++$ & $\pm \sim++$ & $+\underset{(<)}{\sim+t}$ & $+\sim+++$ \\
\hline
\end{tabular}

Table 4. The Methionine-diet for 10 days. The peptic cells.

\begin{tabular}{|c|c|c|c|c|c|c|}
\hline \multirow{3}{*}{ Hrs. } & \multicolumn{3}{|c|}{ Total result } & \multirow{2}{*}{\multicolumn{2}{|c|}{$\begin{array}{l}\text { Representative } \\
\text { result }\end{array}$}} & \multirow{3}{*}{$\begin{array}{l}\text { Gland } \\
\text { lumen }\end{array}$} \\
\hline & \multicolumn{2}{|c|}{$\begin{array}{l}\text { Quantity of the intracel- } \\
\text { lular secretory substance }\end{array}$} & \multirow{2}{*}{$\begin{array}{l}\text { Form of } \\
\text { pls. }\end{array}$} & & & \\
\hline & Granules & Vacuoles & & Granules & Vacuoles & \\
\hline Before & $-\sim++$ & $\pm \sim+(+)$ & $\begin{array}{l}\text { short rod, } \\
\text { long rod, } \\
\text { rosary form, } \\
\text { supra-short } \\
\text { rod, guitar } \\
\text { form }\end{array}$ & $\pm \sim+$ & $\pm \sim+(+)$ & $\begin{array}{l}\text { narrow, } \\
\text { a little wide }\end{array}$ \\
\hline$\frac{1}{2}$ & $-\sim+$ & $-\sim+$ & $\begin{array}{l}\text { short or long } \\
\text { rod, rosary } \\
\text { form, guitar } \\
\text { form }\end{array}$ & $\pm \sim+$ & $-\sim+$ & $\begin{array}{c}\text { a little wide, } \\
\text { narrow }\end{array}$ \\
\hline 1 & $-\sim+++$ & $-\sim \vdash$ & $\begin{array}{l}\text { short or long } \\
\text { rod, rosary } \\
\text { form, guitar } \\
\text { form, supra- } \\
\text { short rod }\end{array}$ & $\pm \sim+$ & $-\sim+$ & $\begin{array}{l}\text { a little wide, } \\
\text { wide, } \\
\text { narrow }\end{array}$ \\
\hline $1 \frac{1}{2}$ & $-\sim+$ & $-\sim \dot{+}$ & $\begin{array}{l}\text { short rod, } \\
\text { long rod, } \\
\text { rosary for } m, \\
\text { guitar form }\end{array}$ & $-\sim+$ & $-\sim+$ & $\begin{array}{c}\text { a little wide, } \\
\text { narrow }\end{array}$ \\
\hline 2 & $-\sim+$ & $-\sim+$ & $\begin{array}{l}\text { long rod, } \\
\text { short rod, } \\
\text { guitar form }\end{array}$ & \pm & $\pm \sim+$ & $\begin{array}{l}\text { narrow, } \\
\text { a little wide }\end{array}$ \\
\hline 3 & $-\sim \pm$ & $-\sim+$ & $\begin{array}{l}\text { long rod, } \\
\text { short rod, } \\
\text { rosary form }\end{array}$ & $-\sim \pm$ & $\pm \sim+$ & $\begin{array}{l}\text { narrow, } \\
\text { a little wide }\end{array}$ \\
\hline
\end{tabular}

Table. 5. The Methionine-diet for 10 days. The productin vacuoles in the gastric surface cells.

\begin{tabular}{c|c|c|c|c|c|c}
\hline & \multicolumn{5}{|c}{ Hrs. } \\
\cline { 2 - 5 } & Before & $\frac{1}{2}$ & 1 & $1 \frac{1}{2}$ & 2 & 3 \\
\hline Total result & $-\sim+++$ & $-\sim++(+)$ & $-\sim++(+)$ & $-\sim+++$ & $\pm \sim+++ \pm \sim+++$ \\
\hline $\begin{array}{c}\text { Representative } \\
\text { result }\end{array}$ & $+\sim+++$ & $+\sim++$ & $+\sim++$ & $+\sim++(+)$ & $\pm \sim+++ \pm \sim+++$
\end{tabular}


Table 6. The Histidine-diet for 10 days. The peptic cells.

\begin{tabular}{|c|c|c|c|c|c|c|}
\hline \multirow{3}{*}{ Hrs. } & \multicolumn{3}{|c|}{ Total result } & \multirow{2}{*}{\multicolumn{2}{|c|}{$\begin{array}{c}\text { Representative } \\
\text { result }\end{array}$}} & \multirow{3}{*}{$\begin{array}{l}\text { Gland } \\
\text { lumen }\end{array}$} \\
\hline & \multicolumn{2}{|c|}{$\begin{array}{l}\text { Quantity of the intracel- } \\
\text { lular secretory substance }\end{array}$} & \multirow{2}{*}{$\begin{array}{l}\text { Form of } \\
\text { pls. }\end{array}$} & & & \\
\hline & Granules & Vacuoles & & Granules & Vacuoles & \\
\hline Before & $-\sim++$ & $\pm \sim++$ & $\begin{array}{l}\text { short rod, } \\
\text { long rod, } \\
\text { rosary form }\end{array}$ & $\pm \sim++$ & $\pm \sim++$ & $\begin{array}{l}\text { narrow, } \\
\text { a little wide }\end{array}$ \\
\hline$\frac{1}{2}$ & $\pm \sim+++$ & $\pm \sim+++$ & $\begin{array}{l}\text { short rod, } \\
\text { guitar form, } \\
\text { rosary form, } \\
\text { long rod }\end{array}$ & $\begin{array}{c}+\sim+t \\
\pm\end{array}$ & $\begin{array}{c} \pm \sim++ \\
+\stackrel{+}{+}+++\end{array}$ & $\begin{array}{l}\text { wide, } \\
\text { a little wide }\end{array}$ \\
\hline 1 & $\pm \sim+++$ & $\pm \sim++$ & $\begin{array}{l}\text { short rod, } \\
\text { supra-short } \\
\text { rod, guitar } \\
\text { form, short } \\
\text { or long ro- } \\
\text { sary form }\end{array}$ & $+\sim++$ & $\pm \sim+$ & $\begin{array}{l}\text { wide, } \\
\text { a little wide }\end{array}$ \\
\hline $1 \frac{1}{2}$ & $-\sim++$ & $\pm \sim++(+)$ & $\begin{array}{l}\text { supra-short } \\
\text { rod, short } \\
\text { rod, guitar } \\
\text { form, (long } \\
\text { rod) }\end{array}$ & $\begin{array}{c}-\sim+ \\
(+\sim++\end{array}$ & $\begin{array}{l}+\sim++(+) \\
\pm \sim+)\end{array}$ & a little wide \\
\hline 2 & $-\sim++$ & $\pm \sim++$ & $\begin{array}{l}\text { short rod, } \\
\text { rosary form, } \\
\text { long rod }\end{array}$ & $-\sim+$ & $\pm \sim+(+)$ & $\begin{array}{l}\text { a little wide, } \\
\text { narrow }\end{array}$ \\
\hline 3 & $-\sim+(+)$ & $-\sim++$ & $\begin{array}{l}\text { long or short } \\
\text { rod, rosary } \\
\text { form }\end{array}$ & $-\sim+$ & $\frac{ \pm \sim+}{(+)}$ & $\begin{array}{l}\text { a little wide, } \\
\text { narrow }\end{array}$ \\
\hline
\end{tabular}

Table 7. The Histidine-diet for 10 days. The productin vacuoles in the gastric surface cells.

\begin{tabular}{c|c|c|c|c|c|c}
\hline & \multicolumn{5}{|c}{ Hrs. } \\
\cline { 2 - 5 } & Before & $\frac{1}{2}$ & 1 & $1 \frac{1}{2}$ & 2 & 3 \\
\hline Total result & $-\sim+++$ & $-\sim+++$ & $-\sim++(+)$ & $-\sim+++ \pm \sim+++ \pm \sim+++$ \\
\hline $\begin{array}{c}\text { Representative } \\
\text { result }\end{array}$ & $\pm \sim+++$ & $\pm \sim++$ & $-\sim++$ & $\pm \sim++| \pm \sim+++|+\sim+++$
\end{tabular}

Table 8. The Tyrosine-diet for 10 days. The peptic cells.

\begin{tabular}{|c|c|c|c|c|c|c|}
\hline \multirow{3}{*}{ Hrs. } & \multicolumn{3}{|c|}{ Total result } & \multirow{2}{*}{\multicolumn{2}{|c|}{$\begin{array}{l}\text { Representative } \\
\text { result }\end{array}$}} & \multirow{3}{*}{$\begin{array}{l}\text { Gland } \\
\text { lumen }\end{array}$} \\
\hline & \multicolumn{2}{|c|}{$\begin{array}{l}\text { Quantity of the intracel- } \\
\text { lular secretory substance }\end{array}$} & \multirow{2}{*}{$\begin{array}{l}\text { Form of } \\
\text { pls. }\end{array}$} & & & \\
\hline & Granules & Vacuoles & & Granules & Vacuoles & \\
\hline Before & $-\sim+$ & $\pm \sim++$ & $\begin{array}{l}\text { long rod, } \\
\text { short rod, } \\
\text { guitar form, } \\
\text { rosary form }\end{array}$ & $-\sim+$ & $\pm \sim+$ & $\begin{array}{l}\text { narrow, } \\
\text { a little wide }\end{array}$ \\
\hline
\end{tabular}


Studies on the Effects of Amino-Acid on the Secretory Activity etc.

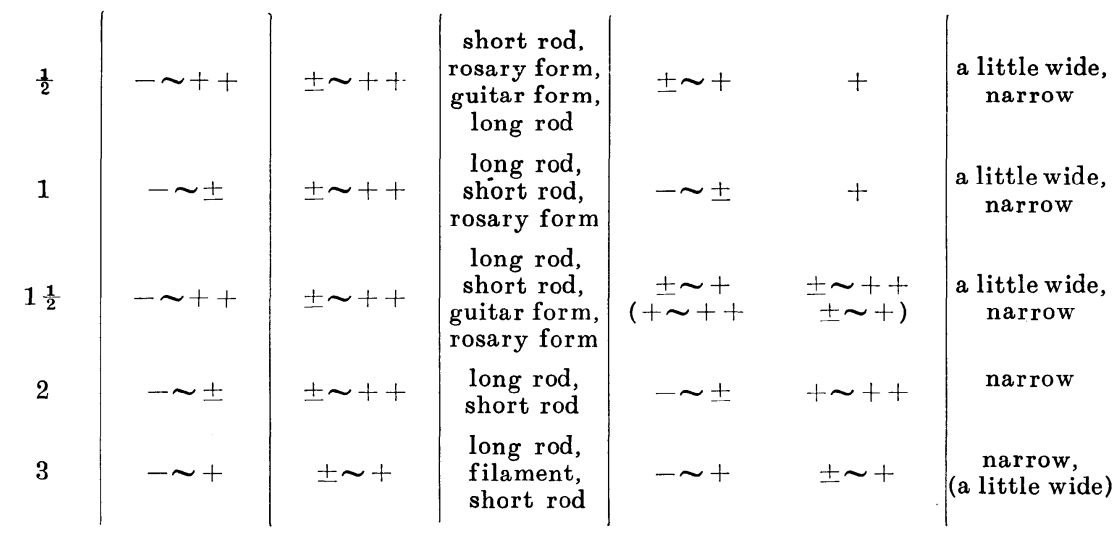

Table 9. The Tyrosine-diet for 10 days. The productin vacuoles in the gastric surface cells.

\begin{tabular}{|c|c|c|c|c|c|c|}
\hline & \multicolumn{6}{|c|}{ Hrs. } \\
\hline & Before & $\frac{1}{2}$ & 1 & $1 \frac{1}{2}$ & 2 & 3 \\
\hline Total result & $\pm \sim+++$ & $\pm \sim+++$ & $\pm \sim+++$ & $\pm \sim+++$ & $\pm \sim+++\mid$ & $+\sim+t+$ \\
\hline esentative result & $+\sim+++$ & $\pm \sim+++$ & $+\sim+++$ & $\pm \sim+++$ & $+\sim+++$ & $+\sim+++$ \\
\hline
\end{tabular}

Table 10. The Tryptophane-diet for 10 days. The peptic cells.

\begin{tabular}{|c|c|c|c|c|c|c|}
\hline \multirow{3}{*}{ Hrs. } & \multicolumn{3}{|c|}{ Total result } & \multirow{2}{*}{\multicolumn{2}{|c|}{$\begin{array}{c}\text { Representative } \\
\text { result }\end{array}$}} & \multirow{3}{*}{$\begin{array}{l}\text { Gland } \\
\text { lumen }\end{array}$} \\
\hline & \multicolumn{2}{|c|}{$\begin{array}{l}\text { Quantity of the intracel- } \\
\text { lular secretory substance }\end{array}$} & \multirow{2}{*}{$\begin{array}{l}\text { Form of } \\
\text { pls. }\end{array}$} & & & \\
\hline & Granules & Vacuoles & & Granules & Vacuoles & \\
\hline Before & $-\sim \pm$ & $\pm \sim+$ & $\begin{array}{l}\text { short rod, } \\
\text { long rod, } \\
\text { rosary form, } \\
\text { guitar form }\end{array}$ & \pm & + & $\begin{array}{l}\text { narrow, } \\
\text { a little wide }\end{array}$ \\
\hline$\frac{1}{2}$ & $-\sim+$ & $\pm \sim++$ & $\begin{array}{l}\text { long or short } \\
\text { rod, guitar } \\
\text { form, supra- } \\
\text { short rod }\end{array}$ & $\pm \sim+$ & $+\underset{(>)}{\sim+}$ & $\begin{array}{c}\text { a little wide, } \\
\text { narrow, } \\
\text { wide }\end{array}$ \\
\hline 1 & $-\sim++$ & $\pm \sim++$ & $\begin{array}{l}\text { short rod, } \\
\text { long rod, } \\
\text { guitar form, } \\
\text { rosary form }\end{array}$ & $-\sim++$ & $\pm \sim++$ & $\begin{array}{c}\text { a little wide, } \\
\text { narrow }\end{array}$ \\
\hline $1 \frac{1}{2}$ & $-\sim+$ & $\pm \sim++$ & $\begin{array}{c}\text { short or long } \\
\text { rod, guitar } \\
\text { form }\end{array}$ & \pm & $+\sim++$ & $\begin{array}{l}\text { a little wide, } \\
\text { narrow }\end{array}$ \\
\hline 2 & $-\sim \pm$ & $\pm \sim++$ & $\begin{array}{c}\text { long rod, } \\
\text { short rod, } \\
\text { rosary form, } \\
\text { guitar form }\end{array}$ & $-\sim \pm$ & $\pm \sim++$ & $\begin{array}{l}\text { a little wide, } \\
\text { wide }\end{array}$ \\
\hline 3 & $-\sim+$ & $\pm \sim++$ & $\begin{array}{l}\text { long or short } \\
\text { rod, guitar } \\
\text { form }\end{array}$ & $-\sim \pm$ & $\pm \sim+$ & a little wide \\
\hline
\end{tabular}


Table 11. The Tryptophane-diet for 10 days. The productin vacuoles in the gastric surface cells.

\begin{tabular}{|c|c|c|c|c|c|c|}
\hline & \multicolumn{6}{|c|}{ Hrs. } \\
\hline & Before & $\frac{1}{2}$ & 1 & $1 \frac{1}{2}$ & 2 & 3 \\
\hline Total result & $\pm \sim+++$ & $\pm \sim+++$ & $\pm \sim+++$ & $-\sim+++$ & $\pm \sim+++$ & $\pm \sim+++$ \\
\hline sentative result & $+\sim+++$ & $\pm \sim+++$ & $\pm \sim+++$ & $\pm \sim+++$ & $+\sim+++$ & $+\sim+++$ \\
\hline
\end{tabular}

Table 12. The Histamine-diet for 10 days. The peptic cells.

\begin{tabular}{|c|c|c|c|c|c|c|}
\hline \multirow{3}{*}{ Hrs. } & \multicolumn{3}{|c|}{ Total result } & \multirow{2}{*}{\multicolumn{2}{|c|}{$\begin{array}{l}\text { Representative } \\
\text { result }\end{array}$}} & \multirow{3}{*}{$\begin{array}{l}\text { Gland } \\
\text { lumen }\end{array}$} \\
\hline & \multicolumn{2}{|c|}{$\begin{array}{l}\text { Quantity of the intracel- } \\
\text { lular secretory substance }\end{array}$} & \multirow{2}{*}{$\begin{array}{l}\text { Form of } \\
\text { pls. }\end{array}$} & & & \\
\hline & Granules & Vacuoles & & Granules & Vacuoles & \\
\hline Before & $-\sim+$ & $\pm \sim++$ & $\begin{array}{c}\text { long rod, } \\
\text { short rod, } \\
\text { long or short } \\
\text { rosary form }\end{array}$ & $-\sim+$ & $+\sim++$ & $\begin{array}{l}\text { a little wide, } \\
\text { narrow }\end{array}$ \\
\hline$\frac{1}{2}$ & $-\sim++$ & $\pm \sim+$ & $\begin{array}{c}\text { short rod, } \\
\text { guitar form, } \\
\text { long rod }\end{array}$ & $\begin{array}{c} \pm \sim+ \\
+\sim++\end{array}$ & $\stackrel{+}{ \pm \sim(+)}$ & $\begin{array}{l}\text { a little wide, } \\
\text { (narrow) }\end{array}$ \\
\hline 1 & $\pm \sim++$ & $\pm \sim++$ & $\begin{array}{l}\text { short rod, } \\
\text { supra-short } \\
\text { rod, guitar } \\
\text { form, rosary } \\
\text { form, long } \\
\text { rod }\end{array}$ & $+\sim++$ & $\pm \sim+(+)$ & a little wide \\
\hline $1 \frac{1}{2}$ & $\pm \sim+++$ & $\pm \sim+++$ & $\begin{array}{l}\text { short rod, } \\
\text { supra-short } \\
\text { rod, guitar } \\
\text { form, short } \\
\text { rosary form }\end{array}$ & $+\sim++(+)$ & $+\sim++$ & $\begin{array}{l}\text { a little wide, } \\
\text { (wide) }\end{array}$ \\
\hline 2 & $\pm \sim++$ & $+\sim+++$ & $\begin{array}{c}\text { short rod, } \\
\text { rosary form, } \\
\text { guitar form, } \\
\text { supra-short } \\
\text { rod, long } \\
\text { rod }\end{array}$ & $\pm \sim++$ & ++ & a little wide \\
\hline 3 & $\pm \sim++$ & $\pm \sim++$ & $\begin{array}{l}\text { long or short } \\
\text { rod, guitar } \\
\text { form }\end{array}$ & $\pm \sim++$ & $\pm \sim++$ & $\begin{array}{l}\text { a little wide, } \\
\text { narrow }\end{array}$ \\
\hline
\end{tabular}

Table 13. The Histamine-diet for 10 days. The productin vacuoles in the gastric surface cells.

\begin{tabular}{|c|c|c|c|c|c|c|}
\hline & \multicolumn{6}{|c|}{ Hrs. } \\
\hline & Before & $\frac{1}{2}$ & 1 & $1 \frac{1}{2}$ & 2 & 3 \\
\hline Total result & $\pm \sim+t+$ & $-\sim++$ & $-\sim+t$ & $-\sim+t+$ & $+\sim+++$ & $\pm \sim+++$ \\
\hline $\begin{array}{c}\text { Representative } \\
\text { result }\end{array}$ & $\pm \sim+++$ & $\pm \sim+++$ & $+\sim++$ & $+\sim++$ & $+(+) \sim+++$ & $t \sim+t+$ \\
\hline
\end{tabular}




\section{B. The pancreatic cells. (Table 14-19)}

In the pancreatic cells, various abnormal changes which may be a result of a lack of protein are observed frequently. These are 1 . the vacuolization of zymogen granules, 2. the vacuoles surrounding the nucleus and 3. the abnormal form of plastosomes such as droplet shaped, gross granule shaped, comma shaped, ring formed, racket shaped or spindle shaped etc. These abnormal structure are identical with those which were previously described by FURUTA (Arch. hist. jap. 11, 1956). However these abnormalities seem to have less affect on the secretory activity of the pancreatic cells, the author has not described them in the tables.

Table 14. The Peptone-diet for 10 days. The pancreatic cells.

\begin{tabular}{|c|c|c|c|c|c|c|c|}
\hline \multirow[b]{2}{*}{ Hrs. } & \multicolumn{3}{|c|}{ Zymogen granules } & \multirow{2}{*}{$\begin{array}{l}\text { Form of } \\
\text { pls. }\end{array}$} & \multirow{2}{*}{$\begin{array}{c}\text { Production } \\
\text { to be } \\
\text { considered }\end{array}$} & \multirow{2}{*}{$\begin{array}{l}\text { Gland } \\
\text { lumen }\end{array}$} & \multirow{2}{*}{$\begin{array}{c}\text { Di scharge } \\
\text { to be } \\
\text { considered }\end{array}$} \\
\hline & Quantity & $\begin{array}{l}\text { Distribu- } \\
\text { tion }\end{array}$ & $\begin{array}{l}\text { Irregulari- } \\
\text { ty in size }\end{array}$ & & & & \\
\hline Before & $+\sim+++$ & loose & little & $\begin{array}{l}\text { short or long } \\
\text { rod, guitar } \\
\text { form }\end{array}$ & $\begin{array}{c}\text { weakest } \\
\text { weak }\end{array}$ & $\begin{array}{c}\text { empty, } \\
\text { straggling, } \\
\text { (massive) }\end{array}$ & weak \\
\hline$\frac{1}{2}$ & $+\sim+++$ & $\begin{array}{l}\text { a little } \\
\text { dense }\end{array}$ & a little & $\begin{array}{l}\text { short rod, } \\
\text { short rosary } \\
\text { form, guitar } \\
\text { form, long } \\
\text { rod }\end{array}$ & $\left.\right|_{\text {middle }} ^{\text {weak }}$ & \begin{tabular}{|} 
straggling, \\
empty, \\
(massive)
\end{tabular} & (middle) \\
\hline 1 & $\pm \sim+(+)$ & & large & $\begin{array}{l}\text { supra-short } \\
\text { rod, short } \\
\text { rod, guitar } \\
\text { form, short } \\
\text { or long } \\
\text { rosary form }\end{array}$ & $\left.\right|_{\text {strong }} ^{\text {middle }}$ & \begin{tabular}{|} 
straggling, \\
massive, \\
blackened, \\
empty
\end{tabular} & $\left.\right|_{\text {strong }} ^{\text {middle }}$ \\
\hline $1 \frac{1}{2}$ & $+\sim++(+)$ & & large & $\begin{array}{l}\text { short rod, } \\
\text { supra-short } \\
\text { rod, guitar } \\
\text { form, long } \\
\text { rod, long } \\
\text { rosary form }\end{array}$ & $\begin{array}{l}\text { strong } \\
\text { middle }\end{array}$ & $\begin{array}{c}\text { straggling, } \\
\text { massive, } \\
\text { blackened, } \\
\text { empty }\end{array}$ & $\underset{\text { strong }}{\operatorname{middle}}$ \\
\hline 2 & $\pm \sim+++$ & loose & $\begin{array}{c}\text { a little } \\
\text { large }\end{array}$ & $\begin{array}{l}\text { short rod, } \\
\text { supra-short } \\
\text { rod, rosary } \\
\text { form, guitar } \\
\text { form, long } \\
\text { rod }\end{array}$ & $\begin{array}{l}\text { strong } \\
\text { middle }\end{array}$ & $\begin{array}{c}\text { straggling, } \\
\text { massive, } \\
\text { blackened, } \\
\text { empty }\end{array}$ & $\underset{\text { strong }}{\operatorname{middle}}$ \\
\hline 3 & $\begin{array}{l}+(+) \\
\quad \sim+-+\end{array}$ & $\begin{array}{l}\text { a little } \\
\text { loose }\end{array}$ & large & $\begin{array}{l}\text { short rod, } \\
\text { guitar form, } \\
\text { long rod, } \\
\text { supra-short } \\
\text { rod }\end{array}$ & $\left.\right|_{\text {(strong) }} ^{\text {middle }}$ & $\begin{array}{c}\text { straggling. } \\
\text { empty, } \\
\text { massive }\end{array}$ & $\left.\right|_{\text {middle }} ^{\text {weak }}$ \\
\hline
\end{tabular}


Table 15. The Methionine diet for 10 days. The pancreatic cells.

\begin{tabular}{|c|c|c|c|c|c|c|c|}
\hline \multirow[b]{2}{*}{ Hrs. } & \multicolumn{3}{|c|}{ Zymogen granules } & \multirow{2}{*}{$\begin{array}{l}\text { Form of } \\
\text { pls. }\end{array}$} & \multirow{2}{*}{$\begin{array}{l}\text { Production } \\
\text { to be } \\
\text { considered }\end{array}$} & \multirow{2}{*}{$\begin{array}{l}\text { Gland } \\
\text { lumen }\end{array}$} & \multirow{2}{*}{$\begin{array}{l}\text { Discharge } \\
\text { to be } \\
\text { considered }\end{array}$} \\
\hline & Quantity & $\begin{array}{l}\text { Distribu- } \\
\text { tion }\end{array}$ & $\begin{array}{c}\text { Irregulari- } \\
\text { ty in size }\end{array}$ & & & & \\
\hline Before & $+\sim+++$ & & a little & $\begin{array}{l}\text { short rod, } \\
\text { long rod, } \\
\text { supra-short } \\
\text { rod, rosary } \\
\text { form }\end{array}$ & weak & $\begin{array}{c}\text { empty, } \\
\text { straggling }\end{array}$ & $\left.\right|_{\text {weak }} ^{\text {weakest }}$ \\
\hline$\frac{1}{2}$ & $\pm \sim++$ & $\begin{array}{l}\text { a little } \\
\text { loose }\end{array}$ & large & $\begin{array}{l}\text { short rod, } \\
\text { guitar form, } \\
\text { rosary form, } \\
\text { long rod }\end{array}$ & (middle) & $\begin{array}{c}\text { empty, } \\
\text { straggling, } \\
\text { massive }\end{array}$ & $\left.\right|_{\text {middle }} ^{\text {weak }}$ \\
\hline 1 & $+\sim+++$ & loose & a little & $\begin{array}{l}\text { short rod, } \\
\text { long rod, } \\
\text { guitar form, } \\
\text { rosary form }\end{array}$ & weak & $\begin{array}{c}\text { empty, } \\
\text { straggling, } \\
\text { (massive) }\end{array}$ & weak \\
\hline $1 \frac{1}{2}$ & $\pm \sim+(+)$ & & a little & $\begin{array}{l}\text { long rod, } \\
\text { short rod, } \\
\text { guitar form, } \\
\text { supra-short } \\
\text { rod, rosary } \\
\text { form }\end{array}$ & weak & $\begin{array}{c}\text { straggling, } \\
\text { massive, } \\
\text { blackened, } \\
\text { empty }\end{array}$ & $\left.\right|_{\text {strong }} ^{\text {middle }}$ \\
\hline 2 & $-\sim++$ & & $\underset{\text { large }}{\text { a little }}$ & $\begin{array}{l}\text { short or long } \\
\text { rod, rosary } \\
\text { form, guitar } \\
\text { form }\end{array}$ & weak & $\begin{array}{c}\text { straggling, } \\
\text { empty, } \\
\text { massive }\end{array}$ & middle \\
\hline 3 & $-\sim+(+)$ & loose & & $\begin{array}{l}\text { long or short } \\
\text { rod, rosary } \\
\text { form }\end{array}$ & $\begin{array}{c}\text { weak } \\
\text { weakest }\end{array}$ & $\begin{array}{c}\text { empty, } \\
\text { straggling }\end{array}$ & $\stackrel{\text { Ieak }}{\text { weakt }}_{\text {weak }}^{\text {to }}$ \\
\hline
\end{tabular}

Table. 16. The Histidine-diet for 10 days. The pancreatic cells.

\begin{tabular}{|c|c|c|c|c|c|c|c|}
\hline \multirow[b]{2}{*}{ Hrs. } & \multicolumn{3}{|c|}{ Zymogen granules } & \multirow{2}{*}{$\begin{array}{l}\text { Form of } \\
\text { pls. }\end{array}$} & \multirow{2}{*}{$\begin{array}{l}\text { Production } \\
\text { to be } \\
\text { considered }\end{array}$} & \multirow{2}{*}{$\begin{array}{l}\text { Gland } \\
\text { lumen }\end{array}$} & \multirow{2}{*}{$\begin{array}{l}\text { Discharge } \\
\text { to be } \\
\text { considered }\end{array}$} \\
\hline & Quantity & $\begin{array}{l}\text { Distribu- } \\
\text { tion }\end{array}$ & $\begin{array}{l}\text { Irregulari- } \\
\text { ty in size }\end{array}$ & & & & \\
\hline Before & $+\sim+++$ & & little & $\begin{array}{l}\text { long rod, } \\
\text { short rod }\end{array}$ & $\begin{array}{c}\text { weakest } \\
\text { weak }\end{array}$ & $\begin{array}{c}\text { empty, } \\
\text { straggling }\end{array}$ & $\begin{array}{c}\text { weakest } \\
\text { weak }\end{array}$ \\
\hline$\frac{1}{2}$ & $\stackrel{+(+) \sim}{++}$ & dense & large & $\begin{array}{l}\text { short rod, } \\
\text { guitar form, } \\
\text { long rod, } \\
\text { supra-short } \\
\text { rod, rosary } \\
\text { form }\end{array}$ & middle & empty, & $\left.\right|_{\text {weak }} ^{\text {weakest }}$ \\
\hline 1 & $+\sim+++$ & & large & $\begin{array}{l}\text { short rod, } \\
\text { rosary form, } \\
\text { guitar form, } \\
\text { long rod }\end{array}$ & (weak) & $\begin{array}{c}\text { straggling, } \\
\text { empty, } \\
\text { massive }\end{array}$ & middle \\
\hline $1 \frac{1}{2}$ & $\pm \sim+++$ & & a little & $\begin{array}{l}\text { short rod, } \\
\text { short rosary } \\
\text { form, guitar } \\
\text { form, supra- } \\
\text { short rod, } \\
\text { long rod }\end{array}$ & middle & $\begin{array}{c}\text { straggling, } \\
\text { empty, } \\
\text { massive }\end{array}$ & middle \\
\hline
\end{tabular}


Studies on the Effects of Amino-Acid on the Secretory Activity etc.

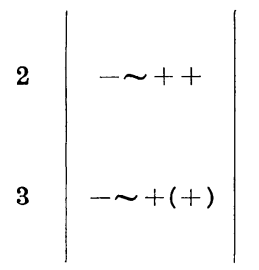

\begin{tabular}{|c|c|c|c|c|}
\hline $\begin{array}{c}\text { a little } \\
\text { large }\end{array}$ & $\begin{array}{l}\text { short rod, } \\
\text { guitar form, } \\
\text { rosary form, } \\
\text { long rod }\end{array}$ & $\begin{array}{c}\text { middle } \\
\text { weak }\end{array}$ & $\begin{array}{c}\text { straggling, } \\
\text { massive, } \\
\text { empty, } \\
\text { blackened }\end{array}$ & $\begin{array}{l}\text { middle } \\
\quad \text { (strong) }\end{array}$ \\
\hline little & $\begin{array}{l}\text { short rod, } \\
\text { long rod, } \\
\text { rosary form, } \\
\text { guitar form }\end{array}$ & weak & $\begin{array}{c}\text { straggling, } \\
\text { massive, } \\
\text { empty }\end{array}$ & middle \\
\hline
\end{tabular}

Table 17. The Tyrosine-diet for 10 days. The pancreatic cells.

\begin{tabular}{|c|c|c|c|c|c|c|c|}
\hline \multirow[b]{2}{*}{ Hrs. } & \multicolumn{3}{|c|}{ Zymogen granules } & \multirow{2}{*}{$\begin{array}{l}\text { Form of } \\
\text { pls. }\end{array}$} & \multirow{2}{*}{$\begin{array}{c}\text { Production } \\
\text { to be } \\
\text { considered }\end{array}$} & \multirow{2}{*}{$\begin{array}{l}\text { Gland } \\
\text { lumen }\end{array}$} & \multirow{2}{*}{$\begin{array}{l}\text { Discharge } \\
\text { to be } \\
\text { considered }\end{array}$} \\
\hline & Quantity & $\begin{array}{l}\text { Distribu- } \\
\text { tion }\end{array}$ & $\begin{array}{c}\text { Irregulari- } \\
\text { ty in size }\end{array}$ & & & & \\
\hline Before & $\pm \sim++$ & & a little & $\begin{array}{l}\text { long rod, } \\
\text { short rod, } \\
\text { rosary form }\end{array}$ & $\left.\right|_{\text {weak }} ^{\text {weakest }}$ & $\begin{array}{c}\text { empty, } \\
\text { straggling }\end{array}$ & $\begin{array}{c}\text { weakest } \\
\text { weak }\end{array}$ \\
\hline$\frac{1}{2}$ & $\pm \sim++$ & & a little & $\begin{array}{l}\text { short rod, } \\
\text { guitar form, } \\
\text { long rod }\end{array}$ & weak & $\begin{array}{c}\text { empty, } \\
\text { straggling }\end{array}$ & $\begin{array}{c}\text { weakest } \\
\text { weak }\end{array}$ \\
\hline 1 & $-\sim++$ & & a little & $\begin{array}{l}\text { short rod, } \\
\text { long rod, } \\
\text { guitar form }\end{array}$ & weak & $\begin{array}{c}\text { straggling, } \\
\text { empty, } \\
\text { massive }\end{array}$ & middle \\
\hline $1 \frac{1}{2}$ & $-\sim++$ & & little & $\begin{array}{l}\text { long rod, } \\
\text { rosary form, } \\
\text { short rod }\end{array}$ & weak & $\begin{array}{c}\text { empty, } \\
\text { straggling }\end{array}$ & $\begin{array}{c}\text { weak } \\
\text { weakest }\end{array}$ \\
\hline 2 & $-\sim++$ & & little & $\begin{array}{l}\text { short rod, } \\
\text { long rod, } \\
\text { guitar form, } \\
\text { rosary form }\end{array}$ & weak & $\begin{array}{c}\text { empty, } \\
\text { straggling, } \\
\text { (massive) }\end{array}$ & weak \\
\hline 3 & $-\sim+(+)$ & loose & little & $\begin{array}{l}\text { long rod, } \\
\text { short rod }\end{array}$ & weakest & $\begin{array}{c}\text { empty, } \\
\text { straggling }\end{array}$ & $\begin{array}{c}\text { weakest } \\
\text { weak }\end{array}$ \\
\hline
\end{tabular}

Table. 18. The Tryptophane-diet for 10 days. The pancreatic cells.

\begin{tabular}{|c|c|c|c|c|c|c|c|}
\hline \multirow{2}{*}{ Hrs. } & \multicolumn{3}{|c|}{ Zy mogen granules } & \multirow{2}{*}{$\begin{array}{l}\text { Form of } \\
\text { pls. }\end{array}$} & \multirow{2}{*}{$\begin{array}{l}\text { Production } \\
\text { to be } \\
\text { considered }\end{array}$} & \multirow{2}{*}{$\begin{array}{l}\text { Gland } \\
\text { lumen }\end{array}$} & \multirow{2}{*}{$\begin{array}{l}\text { Discharge } \\
\text { to be } \\
\text { considered }\end{array}$} \\
\hline & Quantity & $\begin{array}{l}\text { Distribu- } \\
\text { tion }\end{array}$ & $\begin{array}{l}\text { Irregulari- } \\
\text { ty in size }\end{array}$ & & & & \\
\hline Before & $\pm \sim+(+)$ & & & $\begin{array}{l}\text { long or short } \\
\operatorname{rod}\end{array}$ & weakest & $\begin{array}{c}\text { empty, } \\
\text { straggling, } \\
\text { massive }\end{array}$ & $\begin{array}{l}\text { weak } \\
\text { middle }\end{array}$ \\
\hline$\frac{1}{2}$ & $+\sim+++$ & & a little & $\begin{array}{l}\text { short rod, } \\
\text { long rod, } \\
\text { supra-short } \\
\text { rod, guitar } \\
\text { form }\end{array}$ & $\stackrel{l}{\text { weak }}_{\text {(middle) }}$ & $\begin{array}{c}\text { empty, } \\
\text { straggling }\end{array}$ & $\left.\right|_{\text {weakest }} ^{\text {weak }}$ \\
\hline 1 & $\pm \sim++(+)$ & & a little & $\begin{array}{l}\text { long rod, } \\
\text { short rod, } \\
\text { rosary form }\end{array}$ & weak & $\begin{array}{c}\text { empty, } \\
\text { straggling, } \\
\text { (massive) }\end{array}$ & weak \\
\hline $1 \frac{1}{2}$ & $-\sim++$ & & a little & $\begin{array}{l}\text { long rod, } \\
\text { short rod, } \\
\text { rosary form }\end{array}$ & weak & $\begin{array}{c}\text { straggling, } \\
\text { empty }\end{array}$ & weak \\
\hline
\end{tabular}




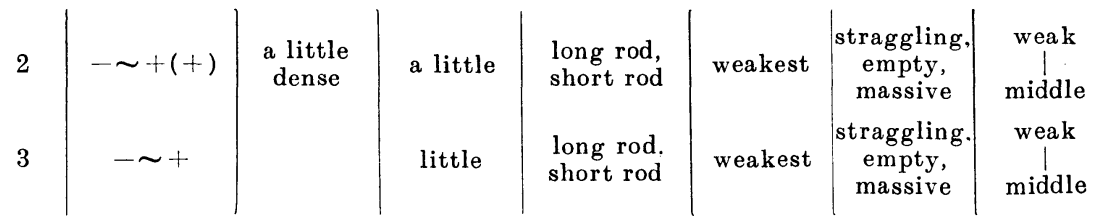

Table 19. The Histamine-diet for 10 days. The pancreatic cells.

\begin{tabular}{|c|c|c|c|c|c|c|c|}
\hline \multirow[b]{2}{*}{ Hrs. } & \multicolumn{3}{|c|}{ Zymogen granules } & \multirow{2}{*}{$\begin{array}{l}\text { Form of } \\
\text { pls. }\end{array}$} & \multirow{2}{*}{$\begin{array}{c}\text { Production } \\
\text { to be } \\
\text { considered }\end{array}$} & \multirow{2}{*}{$\begin{array}{l}\text { Gland } \\
\text { lumen }\end{array}$} & \multirow{2}{*}{$\begin{array}{l}\text { Discharge } \\
\text { to be } \\
\text { considered }\end{array}$} \\
\hline & Quantity & $\begin{array}{l}\text { Distribu- } \\
\text { tion }\end{array}$ & $\begin{array}{l}\text { Irregulari- } \\
\text { ty in size }\end{array}$ & & & & \\
\hline Before & $+\sim+t+$ & loose & & $\begin{array}{l}\text { long rod, } \\
\text { short rod, } \\
\text { rosary form }\end{array}$ & weak & $\begin{array}{c}\text { empty, } \\
\text { straggling }\end{array}$ & $\begin{array}{c}\text { weakest } \\
\text { weak }\end{array}$ \\
\hline$\frac{1}{2}$ & $\pm \sim+t+$ & & a little & $\begin{array}{l}\text { long rod, } \\
\text { short rod, } \\
\text { rosary form, } \\
\text { guitar form }\end{array}$ & weak & $\begin{array}{l}\text { empty, } \\
\text { straggling, } \\
\text { massive }\end{array}$ & middle \\
\hline 1 & $\pm \sim+t$ & loose & a little & $\begin{array}{l}\text { short rod, } \\
\text { short rosary } \\
\text { form, long } \\
\text { rod, guitar } \\
\text { form }\end{array}$ & middle & $\begin{array}{l}\text { straggling, } \\
\text { massive, } \\
\text { empty, } \\
\text { blackened }\end{array}$ & $\begin{array}{c}\underset{\mid}{\text { middle }} \\
\text { (strong) }\end{array}$ \\
\hline $1 \frac{1}{2}$ & $\pm \sim+++$ & & large & $\begin{array}{l}\text { short rod, } \\
\text { guitar form, } \\
\text { supra short } \\
\text { rod, rosary } \\
\text { form, long } \\
\text { rod }\end{array}$ & $\begin{array}{c}\underset{\mid}{\text { middle }} \\
\text { (strong) }\end{array}$ & \begin{tabular}{|} 
straggling, \\
empty, \\
massive
\end{tabular} & $\underset{\text { weak }}{\text { middle }}$ \\
\hline 2 & $+\sim+++$ & $\begin{array}{l}\text { a little } \\
\text { dense }\end{array}$ & a little & $\begin{array}{l}\text { short rod, } \\
\text { rosary form. } \\
\text { long rod, } \\
\text { guitar form }\end{array}$ & 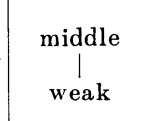 & $\begin{array}{c}\text { straggling, } \\
\text { empty, } \\
\text { massive }\end{array}$ & $\left.\right|_{\text {weak }} ^{\text {middle }}$ \\
\hline 3 & $\pm \sim++(+)$ & & a little & $\begin{array}{l}\text { short rod, } \\
\text { short rosary } \\
\text { form, long } \\
\text { rod, guitar } \\
\text { form }\end{array}$ & weak & $\begin{array}{c}\text { straggling, } \\
\text { massive, } \\
\text { empty }\end{array}$ & middle \\
\hline
\end{tabular}

\section{Discussion.}

For the sake of comparing the secretory activities of the gastric peptic cells with each other and of contrasting them with the previous results obtained by an injection into the stomach, the author described the average quantity of secretion granules and secretion vacuoles in the peptic cells and of productin vacuoles in the gastric surface cells in the same figure (Fig. 1-6).

In the case of the Peptone-diet (Table 2, 3; Fig. 1.), the secretory activity of the peptic cells is similar to that caused by the injection of $10 \%$ Peptone solution into the stomach. On the following two points, there are little differences, viz. 1) the secretion of productin vacuoles after administration of the Peptone-diet seems to be a little weak, 2) secretion vacuoles in the peptic cells are still numerous even at periods of $2-3 \mathrm{hrs}$. after the administration. These may be due to the 


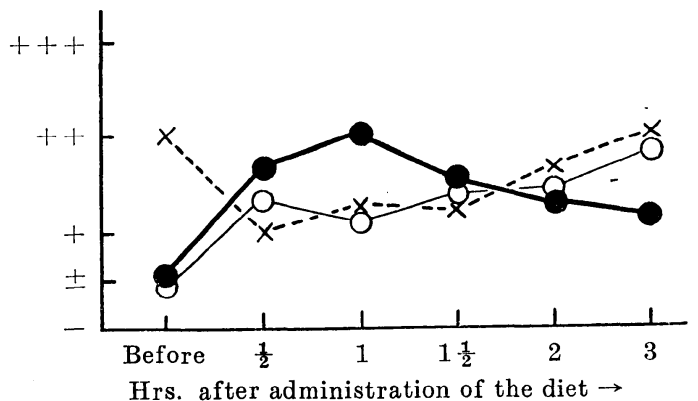

Fig. 1. The Peptone-diet for 10 days. Secretion granules in the peptic cells,

$O$ Secretion vacuoles in them, $\times$ Productin vacuoles in the surface cells.

lesser quantity of Peptone given with the non-protein diet than when its solution was injected into the stomach.

In the case of the Histidine-diet (Table 6, 7; Fig. 2), both discharge of productin vacuoles from the surface cells and production of secretion granules in the peptic cells are very active after the administration but they cease earlier than in

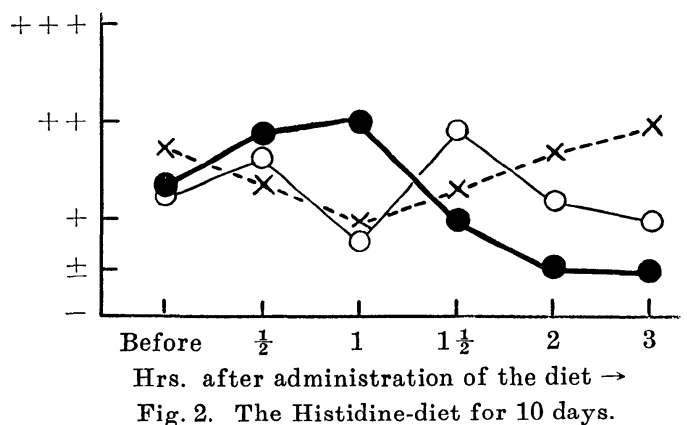

the case where $10 \%$ solution of Histidine was injected into the stomach. This may be due to the quantitative difference of Histidine given. In this case, it is noticed that secretion vacuoles in the peptic cells are numerous and that at periods of $1 \frac{1}{2}$ $-3 \mathrm{hrs}$. after the administration they are identical with those obtained by administration of a normal artificial diet.

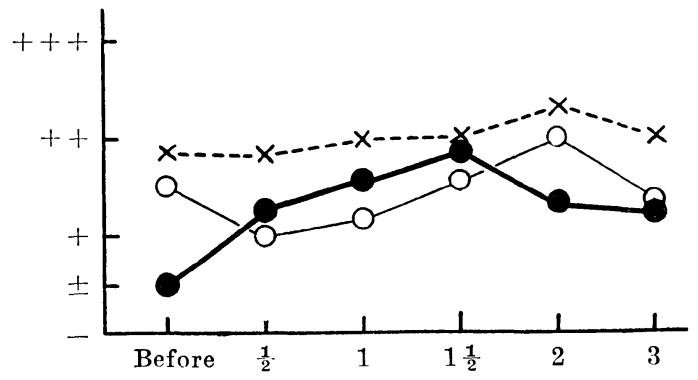

Hrs. after administration of the diet $\rightarrow$

Fig. 3. The Histamine-diet for 10 days. 
In the case of the Histamine-diet (Table 12, 13; Fig. 3), production of secretion granules in the peptic cells grows gradually after the administration, and it becomes as active as in the case where $10 \%$ solution was injected into the stomach. This may indicate that the absorption of Histamine from the wall of the stomach is delayed in this case.

On the other hand, in the case of the Methionine-diet (Table 4, 5; Fig. 4), and of the Tyrosine-diet (Table 8, 9; Fig. 5), and of the Tryptophane-diet (Table 10, 11; Fig. 6), the results are extremely different from those obtained when these amino acicis were injected into the stomach. In case of the Methionine-diet, the lowest

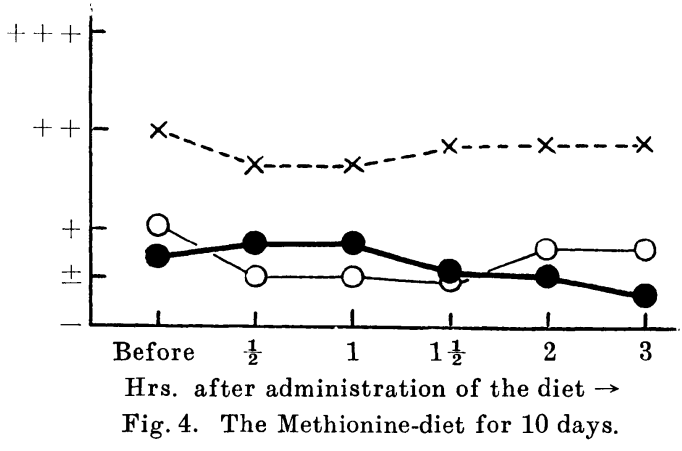

discharge of productin from the gastric surface cells and the lowest production of secretion granules in the peptic cells can be seen. In the case of Tyrosine diet and the Tryptophane-diet, the low discharge of productin and the low production of peptic secretion granules and their relatively active vacuolization are observed. It follows from this result that a small discharge of productin from the surface cells, by which the effect of the decomposed prodnct of the productin can be scarcely seen, will be presumed. The low secretion of productin in these cases is negligible when compared to that obtained by the injection of $10 \%$ solution or emulsion of these amino-acids into the stomach.

As is obvious, Methionine, Tyrosine and Tryptophane which were remarkably effective on the serretory activity of the gastric peptic cells when they were injected into the stomach, have no potency to promote the low secretory activity of the

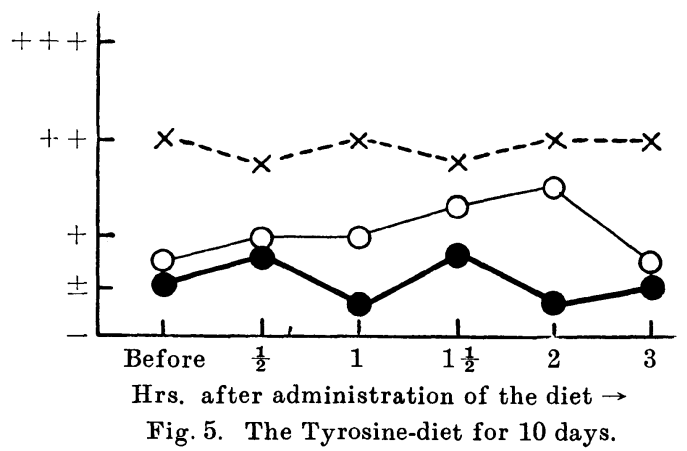




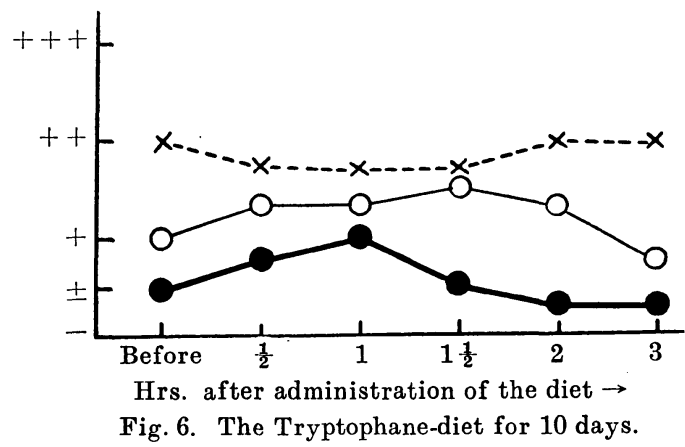

peptic cells caused by a lack of protein in the diet. On the contrary, Histidine and Histamine which were most effective on the secretory activity of the peptic cells after injection into the stomach, have also an effective potency to promote the activity of the cells even if the diet was lacking in protein. As a result of these observations, it is still more probable that to initiat the secretory activity of the gastric peptic cells a substance containing an imidazol group is necessary.

In the pancreatic cells (Table 14-19), the most active secretory function can be seen in the case of the Peptone-diet. In other cases, discharge of zymogen granules is almost the same and is remarkably active. This will be due to the abundance of carbohydrates and the proper quantity of fats contained in the diet. Production of zymogen granules in the cells differs in each case. In the case of the Methionine-diet, of the Tyrosine-diet and of the Tryptophane-diet, it is almost negligible, but in the case of the Histidine-diet and of the Histamine-diet, it is very active. This fact may be the result of the very low secretion of productin in the former three cases, of the active discharge of productin in the Histidine-diet case and of the Histamine absorbed into the blood from the gastric wall in the Histamine-diet case, because the facts that the productin secreted into the blood promotes production of zymogen granules in the pancreatic cells and that an actional similarity between productin and histamine can be seen, have been previously demonstrated by FUJIE in his productin-theory. As is obvious here, the more the substance is effective in discharging productin, the more it promotes the production of zymogen granules in the pancreatic cells, and this is so regardless of administration with the diet or of direct injection into the stomach. Therefore, as regards production of zymogen granules in the pancreatic cells, the fact that humoral stimulation (viz. productin) is still more effective than nervous stimulation will recognized here, as was claimed by FUJIE.

\section{Summary.}

The author proved here, whether the inhibited secretory activity of the gastric peptic and the pancreatic cells caused by a lack of protein in the diet, can be prevented by the amino-acids which are effective in the promotion of the secretory activity of these cells when their solution or emulsion is injected into the stomach of normal rats. The results obtained are as follows. 
1. The only amino-acid which can prevent the inhibited secretion of productin resulting from the feeding with non-protein diet and that can promote the secretory activity (especially production of secretion granules) in the peptic and the pancreatic cells is Histidine.

2. If Histamine is given with a non-protein diet, it does not discharge productin from the gastric surface cells but it sufficiently promotes the secretory activity (especially production of secretion granules) in the peptic and the pancreatic cells. This fact will indicate a similarity between productin and histamine.

3. As a result of these experiments, it can be demonstrated that the initiation of the secretory activity in the gastric peptic and the pancreatic cells requires a substance containing an imidazol group, such as Histidine or Histamine. Here, the fact proved by FUJIE that an imidazol group can be demonstrated histochemically in the subnuclear portion of the gastric surface cells where productin vacuoles are seen, will be of great importance.

\section{内 容 自 抄.}

無蛋白食でラッテを飼育すると胃腺主細胞及び膵細胞の分泌機能が甚だ減退す る事実, その原因は胃腺主細胞や膵細胞自体の機能的退化にあるのでなくて，胃 粘膜表在細胞からの胃壁ホルモン productin 分泌の障害にあると言う実験的証明, 及び微量のヒスチヂン投与がその障害を完全に防止しうる事実等に鑑み，著者は 多くのアミノ酸の10\%溶液又は浮遊液を胃内に注入して検した処. ヒスチヂンの 他にもメチオニン. チロジン, トリプトファンが productin 㕣泌を促し胃腺主細 胞や膵細胞の分泌機能を旺盛ならしめることを知った。依って著者はヒスチヂン, ヒスタミン，メチオニン，チロジン，トリプトファンを夫々無蛋白食に添加し，胃 内注入の時と同様の效果を示すか否かを此所に検した。得たる結果は次の如くで ある。

1. 無蛋白食飼育に由来する productin 分泌障害を防止し, 胃腺主細胞及び膵細 胞の分泌機能（殊に分泌顆粒新生）を促進するアミノ酸はヒスチヂンのみで，メ チオニン，チロジン，トリプトファンは殆ど無効である.

2. ヒスタミン投与は productin を放出させないが, 胃腺主細胞, 膵細胞の分泌 機能（殊に分泌顆籹新生）を活潑ならしめる。此所に於ても productin とヒスタ ミンの相似性が認められる。

3. 上の結果から胃腺主細胞, 膵細胞の分泌機能の営みと imidazol 核とは離す べからざる関係にあることが明らかである。こっに於て productin 空胞の存在部 位に imidazol 核を証明した藤江の組織化学的研究の 結果は誠に意義深いことに なる。

\section{References.}

Esumi, T.: Experimental studies on the relationship between protein-content in diet 
and the function of the gastric gland cells. I, II, III. (Jap. with Engl. abstr.) Arch. hist. jap. 10 (1956). - Fujie, K. et al : Histochemical research on the content in the 'productin' vacuoles (FUJIE) of the gastric surface cells. I and II. Arch. hist. jap. 13 (1957), 14 (1958). - Furuta, K.: Studies on the dietetic characteristics of the secretory functional images in the pancreatic cells. (Jap. with Engl. abstr.) Arch. hist. jap. 11 (1956). - Experimental study on the effects of Histidine in diet to the secretory function of the pancreatic cells. (Jap. with Engl. abstr.) Arch. hist. jap. 12 (1957). - Study on the performing faculty of the secretory function in the pancreatic cells of rats fed with non-protein diet. Arch. hist. jap. 13 (1957). - Nishioka, M.: Studies on the effects of amino-acid on the secretory activity of the gastric peptic cells and the pancreatic cells. Arch. hist. jap. 17 (1959). Tojyo, K.: Experimental studies on the effect of Histidine upon the secretory function of the gastric gland cells. I. Study on Histidine in the diet. Arch. hist. jap. 14 (1958). -- II. On the actional differences between injected Histidine and injected Histamine. Arch. hist. jap. 15 (1958). 